\title{
Sternberg Astronomical Institute Supernova Catalogue, and radial distribution of supernovae in host galaxies
}

\author{
O. S. Bartunov, D. Yu. Tsvetkov, and N. N. Pavlyuk \\ Sternberg Astronomical Institute, Universitetskii pr. 13, 119992 Moscow, Russia
}

\begin{abstract}
We present a new version of the Sternberg Astronomical Institite Supernova Catalogue and the results of our investigation of the supernova radial distribution in their host galaxies based on the new data.
\end{abstract}

Keywords. supernovae: general

We present the results of our ongoing work on statistics of SNe and a new version of SAI SN Catalogue. The previous results and descriptions of earlier versions of the catalogue can be found in Tsvetkov \& Bartunov (1993) and Tsvetkov et al. (2004).

The new catalogue presents an important step towards creation of a modern database on SNe, conforming with the principles of the Virtual Observatory and allowing for new methods for the organization of SN research.

The new investigation of SN radial distributions reveals the following conclusions:

- There is a decrease of SNe Ia density in the central parts of spiral galaxies, which is not observed in ellipticals.

- $\mathrm{SNe} \mathrm{Ib} / \mathrm{c}$ show a high concentration towards the centers of spiral galaxies. Their distribution is different from that for SNe II, in spite of the generally accepted similarity of masses and ages of their progenitors.

- In the outer parts of the spiral galaxies the distributions of SNe ,Ia and SNe II are very similar.

These results are in general agreement with the previous works by van den Bergh (1997), Wang et al. (1997), and Tsvetkov et al. (2004) We should note that they still lack a consistent interpretation and present some difficulties for current theories on the nature of progenitors for different types of SNe.

The complete version of this paper is available at < http://www.sai.msu.su/sn/pubs/sncatdistr.pdf >.

\section{Acknowledgements}

This work was partly supported by the grants 05-02-17480 and 05-07-90225 of the Russian Foundation for Basic Research.

\section{References}

Tsvetkov, D. Yu., \& Bartunov, O. S. 1993, Bull. Inform. CDS 42, 17

Tsvetkov, D. Yu, Pavlyuk, N. N., \& Bartunov, O. S. 2004, Astron. Lett. 30, 729

van den Bergh, S. 1997, AJ, 113, 197

Wang, L., Hoflich, P., \& Wheeler, J. C. 1997, ApJ (Letters), 483, L29 